\title{
Human Hippocampal Theta Activity During Virtual Navigation
}

\author{
Arne D. Ekstrom, ${ }^{1,2}$ Jeremy B. Caplan, ${ }^{3}$ Emily Ho, ${ }^{2}$ Kirk Shattuck, ${ }^{2}$ \\ Itzhak Fried, ${ }^{2,4^{*}}$ and Michael J. Kahana ${ }^{5^{*}}$
}

\begin{abstract}
This study examines whether $4-8-\mathrm{Hz}$ theta oscillations can be seen in the human hippocampus, and whether these oscillations increase during virtual movement and searching, as they do in rodents. Recordings from both hippocampal and neocortical depth electrodes were analyzed while six epileptic patients played a virtual taxi-driver game. During the game, the patients alternated between searching for passengers, whose locations were random, and delivering them to stores, whose locations remained constant. In both hippocampus and neocortex, theta increased during virtual movement in all phases of the game. Hippocampal and neocortical theta activity were also significantly correlated with each other, but this correlation did not differ between neocortex and hippocampus and within disparate neocortical electrodes. Our findings demonstrate the existence of movement-related theta oscillations in human hippocampus, and suggest that both cortical and hippocampal oscillations play a role in attention and sensorimotor integration. $\odot 2005$ Wiley-Liss, Inc.
\end{abstract}

KEY WORDS: intracranial EEG; theta oscillations; spatial navigation; sensorimotor integration

\section{INTRODUCTION}

The rodent hippocampal theta rhythm is manifest in a variety of behavioral tasks, but it has been most thoroughly studied during spatial navigation. As a rat runs around a track, theta power increases linearly with running speed (Vanderwolf, 1969; McFarland et al., 1975; Reece, 1994; Czurko et al., 1999). This relation between theta power and running speed is not affected by age (Shen et al., 1997) or NMDA blockade (Ekstrom et al., 2001), suggesting that changes in theta oscillations may relate to intrinsic network properties (Bland and Colom, 1993; Kamondi et al., 1998) and may not be as plastic as cellular firing rates. Further evidence suggests that the hippocampal theta rhythm plays a role in the timing of action potentials during place learning (Mehta

${ }^{1}$ Division of Brain Mapping and ${ }^{2}$ Division of Neurosurgery and Department of Psychiatry and Biobehavioral Science, University of California, Los Angeles, California 90095; ${ }^{3}$ The Rotman Research Institute, Baycrest Centre for Geriatric Care, Toronto, Ontario M6A 2E1, Canada; ${ }^{4}$ Functional Neurosurgery Unit, Tel-Aviv Medical Center and Sackler School of Medicine, Tel-Aviv University, Tel-Aviv 69978, Israel; ${ }^{5}$ Department of Psychology, University of Pennsylvania, Philadelphia, Pennsylvania 19104

*Correspondence to: M. Kahana, Department of Psychology, University of Pennsylvania, Philadelphia,PA 19104. E-mail: kahana@psych.upenn.edu or I. Fried, Division of Neurosurgery and Department of Psychiatry and Biobehavioral Science, University of California, Los Angeles, CA 90095.

E-mail: ifried@mednet.ucla.edu.

Accepted for publication 3 June 2005

DOI 10.1002/hipo.20109

Published online 19 August 2005 in Wiley InterScience (www.interscience. wiley.com). et al., 2002), while place representations themselves ("place cells") are not significantly altered by abolition of the theta rhythm (Leutgeb and Mizumori, 1999). In humans, cortical theta oscillations have also been observed during a variety of learning tasks, including recognition (Raghavachari et al., 2001) and recall (Sederberg et al., 2003), and are typically defined to be in the $4-8-\mathrm{Hz}$ range (Niedermeyer and Lopes da Silva, 1999). Human theta activity has also been shown to increase during virtual spatial navigation tasks (Kahana et al., 1999; Caplan et al., 2001; de Araújo et al., 2002). Caplan et al. (2003) further showed that a greater number of cortical electrodes exhibited increased movement-related theta during searching for spatially variant objects (randomly placed passengers) than during searching for spatially invariant objects (fixed-location stores). The exact role of theta oscillations during learning and spatial navigation, however, remains unclear. The discovery of hippocampal place cells in rats (O'Keefe and Dostrovsky, 1971) and in humans (Ekstrom et al., 2003), and the tight coupling of place-cell firing to the theta rhythm (O'Keefe and Reece, 1993), have supported the idea that theta oscillations are important for place learning, at least in the rodent (Mehta et al., 2002). The relative absence of place cells in the neocortex (Jung et al., 1998) and the coupling of theta, place-cell firing, and spatial learning in the hippocampus suggest that theta oscillations in the hippocampus and cortex ought to serve different functions, and therefore be manifest during different behavioral tasks (see also Green and Arduini, 1954). It has been argued, however, based on the fact that theta oscillations are present in disparate neocortical sites during a variety of cognitive tasks (Berry and Seager, 2001; Caplan et al., 2003), that theta oscillations play a more general role in attention and sensorimotor integration (Komisaruk, 1970; Bland, 1986; Bland and Oddie, 2001).

Our first objective was to test these competing explanations by examining whether theta oscillations are present in the human hippocampus during voluntary movement in a virtual taxi-driver game called "Yellow Cab." In addition to demonstrating movementrelated cortical theta, Caplan et al. (2003) had shown improved route efficiency when subjects searched for fixed-location stores but not when they searched for randomly placed passengers. In their study, learning occurred during searching for stores because their 
location was constant and therefore could be learned. Thus, our second objective was to compare two competing hypotheses: do theta oscillations in the neocortex and hippocampus occur during different epochs of navigation, such as searching for randomly placed passengers versus searching for fixed-location stores, or do theta oscillations in hippocampus and neocortex occur to similar degrees during the same behavioral epochs? Support for the first hypothesis would bolster the idea that hippocampal theta relates to cognitive-map formation, because Caplan et al. (2003) had shown that locations of stores could be learned during navigation. To address these two competing hypotheses, we analyzed oscillations (particularly theta) in hippocampus and cortex (including parahippocampal region and neocortex), using intracranial electroencephalographic (iEEG) recordings in patients undergoing seizure monitoring while they played Yellow Cab.

\section{MATERIALS AND METHODS}

\section{Behavioral Methods}

The behavioral methods are identical to those described in the works of Ekstrom et al. (2003) and Caplan et al. (2003). Briefly, subjects navigated using the four arrow keys on a computer keyboard; when moving, velocity was constant. Virtual cities in Yellow Cab consisted of six unlabeled, nontarget buildings and three labeled, target stores (Fig. 1a). The city was thus made up of three different blocks in both the north-south and east-west directions. During a single session, subjects made seven deliveries of passengers to each target store in a random order. Passengers were picked up by driving near them; text then appeared instructing subjects where to deliver the passenger. A small line of text in the corner of the screen remained present throughout the delivery to remind subjects of their goal. Each delivery began from the random position where the passenger was picked up. On delivery of the passenger to a fixed-location store (accomplished by driving into it), a text message told subjects whether they had found the correct store (subjects also received "virtual" payment for delivering passengers). A text screen instructed subjects to find another passenger, and the subjects explored the city until they located the passenger, at which point the cycle began again.

Stores and passengers looked the same from all angles from which they were viewed; stores were identified by highly visible names. Subjects could not make arcing turns; if more than one arrow key was pressed, only the most recent key-press would apply. The turning rate was $20 \mathrm{deg} / \mathrm{s}$ such that it would take $18 \mathrm{~s}$ to complete a full turn. The walking speed was set to 1.17 blocks/s; the view was refreshed every $30 \mathrm{~ms}$. Periods of movement epochs were determined as times when subjects held down the forward, right, or left arrow keys. Standing still was defined as the absence of any key-presses and did not include times when the subject was reading text instructions. A complete block of Yellow Cab included first exploring one city (A), then exploring a novel city $(B)$, and then returning to $A\left(A^{\prime}\right)$. In some cases we were unable to run more than one or two sessions because of patient seizures or technical issues. Sessions in which a subject made fewer than 19 deliveries were excluded. a.



b.

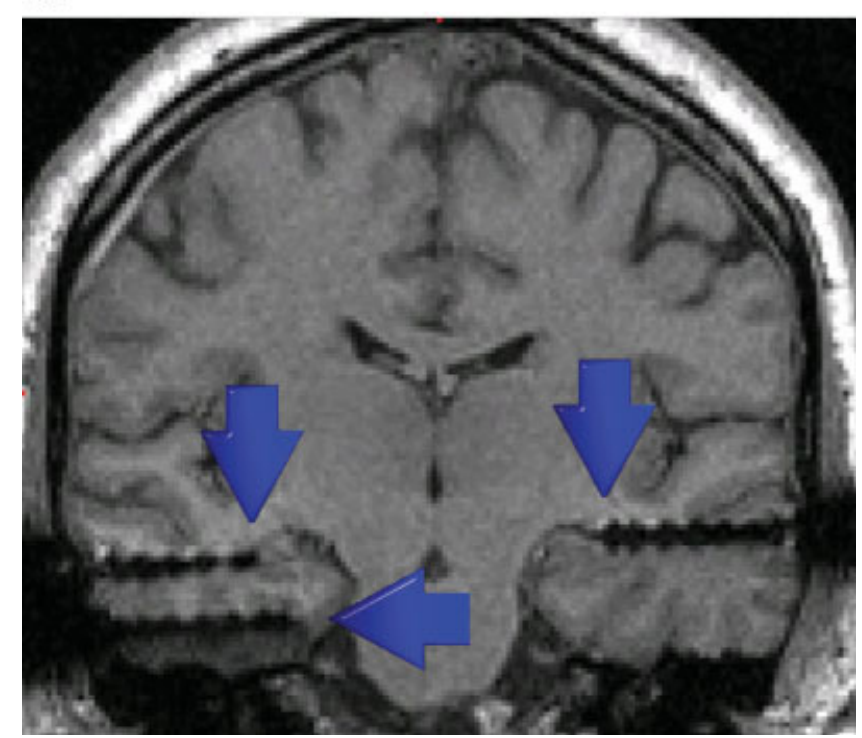

FIGURE 1. Behavioral methods and recordings. (a) Screen shot of the virtual environment that subjects explored. During navigation, subjects moved by pressing the arrow keys. Subjects first searched for randomly placed passengers, and then sought out fixedlocation stores. (b) MRI of depth electrodes directed bilaterally to the hippocampi and unilaterally to the parahippocampal cortex of a patient. Top two bilateral electrodes contact the hippocampus; bottom electrode on left contacts the parahippocampal cortex. [Color figure can be viewed in the online issue, which is available at www. interscience.wiley.com.]

We thus recorded a total of 11 sessions of Yellow Cab. Because we did not observe any differences in electroencephalography (EEG) patterns when exploring different environments (see also Caplan et al., 2003), recordings from different sessions on the same patient were grouped together.

\section{Patient Data and Electrophysiology}

Five (of six) patients were right-handed, and one was female. One patient had right temporal-lobe epilepsy; all others had 
TABLE 1.

Patient Data and Numbers of Included and Excluded Electrodes

\begin{tabular}{ccccccc}
\hline Patient no. & Age & Sex & Handedness & Included sites & Excluded sites & Resection area \\
\hline 1 & 38 & M & R & 45 & 43 & Left medial temporal \\
2 & 46 & M & R & 23 & 39 & Right medial temporal \\
3 & 48 & M & R & 24 & 30 & Left medial temporal \\
4 & 42 & F & L & 26 & 31 & Left medial temporal \\
5 & 41 & M & R & 32 & 28 & Left medial temporal \\
6 & 45 & M & R & 28 & Left medial temporal \\
\hline
\end{tabular}

left temporal-lobe epilepsy. Each patient had between 6 and 14 depth electrodes implanted bilaterally from a lateral orthogonal approach (surgeries were performed by I.F.). EEG signal was recorded using the 128 Telefactor EEG recording system. Magnetic resonance imaging (MRI) scans following placement of electrodes, or postplacement computed tomography (CT) scans coregistered to preoperative MRI scans, were used to verify the anatomical location of all depth-electrode contacts (Fig. 1b); (see also Fried et al., 1997, 1999; Cameron et al., 2001). Neocortical electrodes were contacts localized to temporal, frontal, occipital, and parietal neocortices. Hippocampal recordings were obtained from contacts that terminated in the hippocampus proper. Parahippocampal recordings were obtained from any contact placed in entorhinal cortex, parahippocampal cortex, or the collateral sulcus (Witter and Wouterlood, 2002). "Cortex" in this manuscript therefore includes neocortex, parahippocampal and entorhinal cortex, and collateral sulcus. Electrode contacts placed in areas where seizure activity had occurred were excluded from the analysis; thus, 134 electrode contacts were excluded because they were in seizure areas (see Table 1). Eighty-four electrode contacts were determined, during the process of electrode localization, to be in white matter or bone; these recording sites were also excluded from the analysis. A total of 218 electrodes were excluded across all patients, leaving a total of 147 electrodes in neocortex, 19 in the parahippocampal region, and 13 in hippocampus. See Table 1 for details on patient demography. This study conformed with the guidelines of the UCLA Medical Institutional Review Board. Informed consent was obtained from all patients. The results from single-cell recordings with five (of six) of these same patients during virtual navigation have been reported previously (Ekstrom et al., 2003).

\section{Data Analysis}

We quantified oscillatory episodes using the method of Caplan et al. (2001, 2003). This approach identifies epochs of iEEG signal that show high-oscillatory power at a particular frequency lasting several cycles. The method excludes much of the background noise signal by estimating the noise spectrum. A minimum-duration threshold helps to exclude spikes, sharp waves, and nonrhythmic changes in power. The analysis is per- formed separately at each frequency of interest. For a given frequency, $f^{*}$, an oscillatory episode is defined as an epoch longer than a duration threshold, $D_{\mathrm{T}}$ (in numbers of cycles), during which wavelet power at frequency $f^{*}$ exceeded a power threshold, $P_{\mathrm{T}}$. The two threshold parameters were chosen as follows: (1) We wavelet-transformed the raw iEEG signal [Morlet wavelet, window $=6$ cycles, Grossmann and Morlet (1985)] at 22 logarithmically spaced frequencies in the range $1-32 \mathrm{~Hz}$ to obtain the wavelet power spectrum. (2) We assumed that the background noise spectrum has the form $\operatorname{Power}(f)=A f^{-\alpha}$. We estimated this background by fitting the observed spectrum (at each electrode) with a linear regression in $\log -\log$ units. Because wavelet power values are expected to be distributed like $\chi^{2}(2)$ (Percival and Walden, 1993), the estimated background at $f^{*}$ should be the mean of its corresponding $\chi^{2}(2)$ distribution. We chose $P_{\mathrm{T}}(f)$ to be the 95 th percentile of the fit distribution. Power thresholding should exclude about $95 \%$ of the estimated background signal. (3) We set $D_{\mathrm{T}}$ to three cycles of $f$, or $3 / f$. This was done to eliminate artifacts and nonrhythmic physiological signals. (4) Finally, $P_{\text {episode }}(f)$, or the percentage of time in oscillatory episodes, was defined as the total amount of time filled with detected oscillatory episodes divided by the total time in the segment of interest. In subsequent analyses, we considered the $1-32-\mathrm{Hz}$ range, excluding frequencies at the ends of the spectrum to keep clear of the bandpass filtering of the amplifiers (low end) and the $60-\mathrm{Hz}$ line noise (high end).

We define a traversal within Yellow Cab as a period of time from passenger pick-up to store delivery or from store delivery to passenger pick-up. A single session comprised 42 traversals, half of which involved searching for passengers and half of which involved searching for stores. We next calculated for each traversal the mean $P_{\text {episode }}$ value for moving and standing-still epochs. To determine whether oscillations changed as a result of movement, we performed a Mann-Whitney $U$ test to compare the distribution of $P_{\text {episode }}$ values for moving versus standing still across the different traversals. Data recorded from the same electrodes in different sessions of the game were pooled. A bootstrap technique was used to determine the critical $U$ value signifying whether a given electrode exhibited movementrelated oscillations. This was done by randomly shuffling the labels assigning $P_{\text {episode }}$ values to movement or still epochs 
1,000 times for each subject and calculating the $U$ value for each pseudosession. For each patient, all pseudo- $U$ values were grouped and sorted; $U$ critical values were then selected based on the element in the sorted array that was greater than or equal to the percentile $\alpha$-value chosen for the comparison. Cortical and hippocampal $U$ critical values were computed together. An electrode was deemed to show a significant effect if the $U$ value from the Mann-Whitney test was greater than the $U$ critical value determined by our bootstrapped mean $P_{\text {episode }}$ at an $\alpha$-value of 0.01 , which was used for all electrodeby-electrode comparisons.

\section{RESULTS}

Moving and standing-still epochs were compared for each session completed by each subject. Subjects spent more time moving $(1.80 \pm 0.13 \mathrm{~s})$ than standing still $(1.18 \pm 0.22 \mathrm{~s})$, $t(12)=2.4, P<0.02$. Movement and standing-still epochs, however, were similar in duration whether subjects were searching for passengers or stores, $t(12)=0.2$. In one session, a subject remained still for a total of only $1.83 \mathrm{~s}$ over the entire 24min experiment; thus, that session was not included in the analysis. During virtual movement, theta oscillations could be seen clearly in the raw EEG traces from a number of hippocampal recording sites (Figs. 2a,b). Furthermore, theta power was typically greater during epochs of virtual movement than during epochs of stillness (Figs. 2c,d). Strong theta oscillations were also observed in plots of $P_{\text {episode }}(f)$ values as a function of frequency on these same electrodes (Figs. 2e,f). Data from these hippocampal electrodes reveal a significant increase in theta activity during virtual movement. We next examined whether this movement-related theta effect appeared consistently across the population of electrodes implanted in the hippocampus and cortex (e.g., neocortex and parahippocampal region) across all six patients. For each cortical and hippocampal electrode, we compared oscillatory power during epochs of movement and stillness. These comparisons were made at each frequency between 1 and $32 \mathrm{~Hz}$.

Consistent with the results of Caplan et al. (2003), analyses of neocortical electrodes revealed significant increases in oscillatory power during virtual movement as compared with that while standing still at all frequency bands (Table 2): e.g., moving $>$ standing still, using a Mann-Whitney $U$ test (see Methods). We observed a small number of electrodes in the neocortex in the delta, theta, and alpha bands showing the reverse effect during searching for passengers: e.g., standing still > moving (Table 2). Because these standing still $>$ moving effects were small in number compared with moving $>$ standing still effects (69/147 electrodes showing moving $>$ standing still vs. $9 / 147$ electrodes showing standing still $>$ moving effects, $\chi^{2}(1)=$ 46, $P<0.000001$ ), we do not consider these effects further. We also observed significant hippocampal movement-related oscillations in the delta and alpha range (Table 2). Given that rat hippocampal theta is typically measured from 3 to $12 \mathrm{~Hz}$, we might be justified in including significant electrodes from the upper
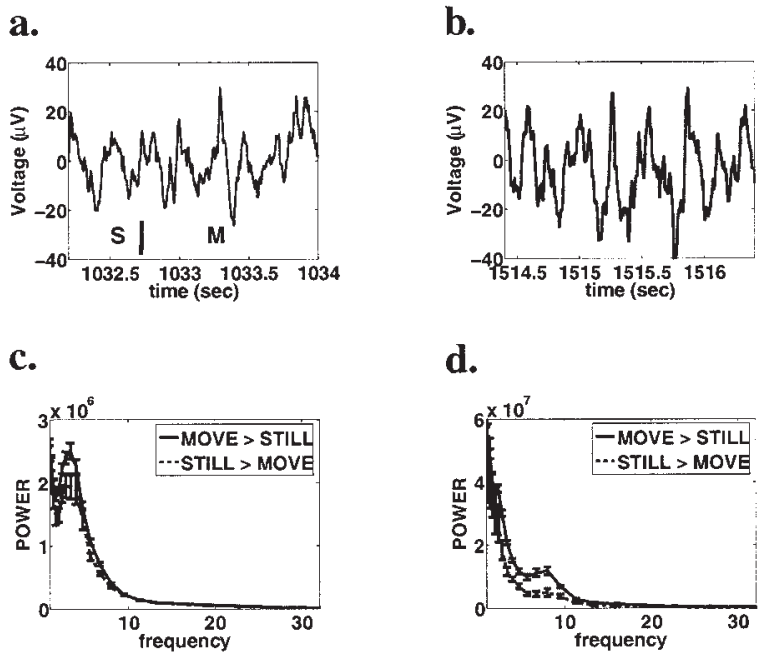

e.



f.



FIGURE 2. Theta during virtual movement. Raw traces from two different electrodes in the right hippocampus of patient no. 1 , showing theta oscillations during virtual movement. Raw trace in (a) shows standing still (S) preceding a movement epoch (M); (b) shows movement only. Power spectrum (c and d) and $P_{\text {episode }}(f)$ (e and f) plots for the same electrode show significant differences in oscillatory power for moving vs. standing still during searching for passengers (a, c, and e) and searching for stores (b, $d$, and $f$ ). Units of power are in $\mu \mathrm{V}^{2} / \mathrm{Hz}$; units of frequency are in $\mathrm{Hz}$.

delta range (e.g., $\geq 3 \mathrm{~Hz})$ or lower alpha range $(\leq 12 \mathrm{~Hz})$. For the sake of consistency with previous work on human cortical theta, however, we restrict our analysis of theta to the more conservative range of $4-8 \mathrm{~Hz}$ (e.g., Niedermeyer and Lopes da Silva, 1999). We note, however, that broadening the theta band to include frequencies classically analyzed in the rat does not affect our overall findings.

To better quantify the number of electrodes in each brain region that showed significant theta band effects, we compared the total number of unique electrodes in the theta band (in hippocampus and cortex) that significantly exceeded the Type I error rate during searching for passengers and searching for stores (see Tables 2 and 3). This was done by comparing the actual number of significant electrodes for passenger searching versus store searching and the expected distribution of an equal number of electrodes in each epoch, based on a $\chi^{2}$ test. In neocortex, significantly more electrodes than would be expected by chance showed movement-related theta effects during searching for passengers when compared with searching for stores (59/ 147 vs. $\left.23 / 147 ; \chi^{2}(1)=15, P<0.0001\right)$. These findings 
TABLE 2.

Number of Significant Electrodes by Frequency Band and Anatomical Region

\begin{tabular}{|c|c|c|c|c|c|}
\hline \multirow[t]{3}{*}{ Neocortex } & Moving $>$ still, passenger & 55 & 59 & 51 & 39 \\
\hline & Moving $>$ still, store & 42 & 23 & 42 & 27 \\
\hline & Still $>$ moving, store & 0 & 0 & 1 & 0 \\
\hline \multirow[t]{2}{*}{ Hippocampus } & Moving $>$ still, passenger & 8 & 5 & 3 & 4 \\
\hline & Still $>$ moving, store & 0 & 0 & 0 & 0 \\
\hline \multirow[t]{4}{*}{ Parahippocampal } & Moving > still, passenger & 9 & 9 & 10 & 9 \\
\hline & Moving $>$ still, store & 4 & 5 & 4 & 5 \\
\hline & Still $>$ moving, passenger & 0 & 0 & 0 & 0 \\
\hline & Still $>$ moving, store & 0 & 0 & 0 & 0 \\
\hline
\end{tabular}

Type I error rate for neocortex is 2 electrodes; for hippocampus and parahippocampal region, 0.2 electrodes. Type I error rate is based on an $\alpha=0.01$, or $1 \%$ of all electrodes in the region.

replicated previous results (Caplan et al., 2003). This effect was significant when we examined regions within the neocortex as well (e.g., temporal cortex, $21 / 51$ vs. $7 / 51, \chi^{2}(1)=7.0, P<$ 0.01 ; and frontal cortex, $35 / 83$ vs. $12 / 83, \chi^{2}(1)=11.2, P<$ 0.001 ; see Table 3$)$. There was no significant difference in the fraction of electrodes that exhibited movement-related theta during searching for passengers versus searching for stores in the parahippocampal region $\left(9 / 18\right.$ vs. $5 / 18 ; \chi^{2}(1)=1.1, P=$ 0.3 ) or hippocampus (5/13 vs. $\left.4 / 13 ; \chi^{2}(1)=0.1, P=0.8\right)$. Combining data from parahippocampal and hippocampal elec- trodes to increase statistical power did not reveal a significant difference in the fraction of electrodes that exhibited movement-related theta during searching for passengers versus searching for stores ( $14 / 31$ vs. $\left.9 / 31 ; \chi^{2}(1)=1.1, P=0.3\right)$. To address whether hippocampal and neocortical sites tended to show the same or different trends in terms of the number of sites showing significant theta oscillations during searching for passengers versus searching for stores, we performed a $\chi^{2}$ bivariate tabular analysis. There was no significant interaction between neocortex, parahippocampal region, and hippocampus

TABLE 3.

Significant Theta $(\theta)$ Effects by Anatomical Subregion

\begin{tabular}{lccc}
\hline Region & $\begin{array}{c}\text { No. of significant } \theta \\
\text { electrodes: searching for passengers }\end{array}$ & $\begin{array}{c}\text { No. of significant } \theta \\
\text { electrodes: searching for stores }\end{array}$ & Total electrodes \\
\hline Temporal cortex & 0 & 0 & 1 \\
Insula & 3 & 1 & 4 \\
Fusiform & 10 & 4 & 14 \\
ITG & 8 & 2 & 28 \\
MTG & 0 & 0 & 4 \\
STG & & & 51 \\
Frontal cortex & 22 & 9 & 9 \\
OF & 3 & 0 & 6 \\
Cingulate & 2 & 0 & 14 \\
IFG & 8 & 3 & 3 \\
MFG & 0 & 0 & 11 \\
SFG & 3 & 4 & 2 \\
Parietal cortex & 0 & 0 & 13 \\
Occipital cortex & 5 & 4 & 18 \\
Hippocampus & 9 & 5 & \\
Parahippocampal & & & \\
\hline
\end{tabular}

ITG, inferior temporal gyrus; MTG, middle temporal gyrus; STG, superior temporal gyrus; OF, orbital frontal cortex; IFG, inferior frontal gyrus; MFG, middle frontal gyrus; SFG, superior frontal gyrus. Columns 1 and 2 indicate the numbers of significant theta electrodes found for each effect (all effects are thus for movement-related theta). 
for the number of electrodes showing movement-related theta during searching for passengers and searching for stores $\left(\chi^{2}(2)=\right.$ $0.8, P=0.5)$. This effect did not become significant when we combined parahippocampal and hippocampal recordings to increase statistical power (14/31 and $9 / 31$ for parahippocampal-hippocampus vs. $59 / 167$ and $23 / 167$ for neocortex; $\left.\chi^{2}(1)=0.7, P=0.5\right)$. The results of our $\chi^{2}$ dissociation analysis demonstrate that there was no significant difference between neocortex and hippocampus for those aspects of behavior that triggered movement-related theta during navigation in our disparate recording sites.

Although our analysis demonstrates that theta oscillations are increased at multiple recording sites in hippocampus and cortex across multiple subjects during movement, and to similar degrees during different aspects of navigation (e.g., searching for passengers vs. searching for stores), it could be that theta oscillations do not covary during navigation. For example, hippocampal theta oscillations may increase during one phase of learning while cortical oscillations increase during a different phase of learning. In an attempt to rule out this possibility, we correlated bouts of theta over learning within electrodes in the neocortex, and then contrasted these correlations with those observed between hippocampal and neocortical electrodes. This analysis was performed in several steps. First, we computed the mean $P_{\text {episode }}(f)$ value in the theta band for each electrode in hippocampus and neocortex separately for each traversal (see Methods). Electrodes that did not show significant movementrelated theta effects in our previous analysis were not considered (see Table 3). For each traversal, subjects searched either for passengers or stores; as before, these two epochs were considered separately. For each electrode, then, a total of 42 $P_{\text {episode }}(f)$ values were tabulated in hippocampus and neocortex, with $21 P_{\text {episode }}(f)$ values for passenger searching and 21 $P_{\text {episode }}(f)$ values for store searching. For every pair of neocortical electrodes that showed significant movement-related theta, we correlated the $P_{\text {episode }}(f)$ values across traversals. We conducted the same analysis for pairs of electrodes in hippocampus and neocortex showing movement-related theta effects. Thus, for the neocortex-neocortex correlations, 59 electrodes were included in the analysis of searching for passengers and 23 electrodes were included in the analysis of searching for stores. For the neocortex-hippocampus correlations, 59 cortical electrodes and 5 hippocampal electrodes were included in the analysis of searching for passengers, and 23 cortical and 4 hippocampal electrodes were included in the analysis of searching for stores. We did not compare correlation coefficients within the hippocampus because we only recorded data from one or two electrodes in hippocampus for any given patient (see Methods and Table 2).

The mean correlation within neocortex was $0.39 \pm 0.01$ for passenger searching and $0.31 \pm 0.02$ for store searching. Between neocortex and hippocampus the correlation was 0.41 \pm 0.02 for passenger searching and $0.31 \pm 0.04$ for store searching (see Fig. 3). To determine whether there was a significant difference or interaction between neocortex and hippocampus, we performed a $2 \times 2$ epoch-by-region analysis of var-

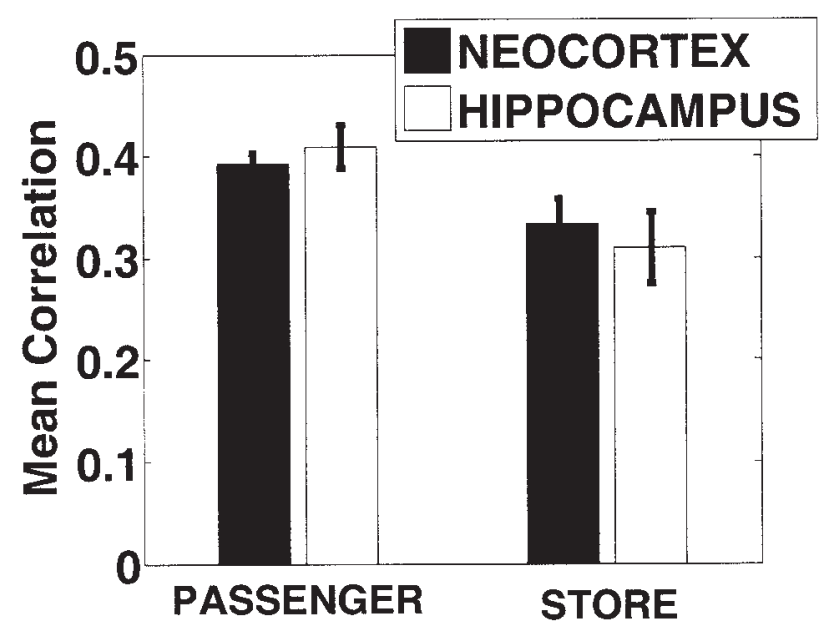

FIGURE 3. Correlation of theta activity both within neocortex and between neocortex and hippocampus The mean correlations over traversals within electrodes in the neocortex and between electrodes in neocortex and hippocampus are shown separately for searching for passengers and searching for stores. Error bars are the standard error of the mean. We did not find a significant dissociation when we compared within-neocortex and neocortex-hippocampus correlation values (see Results).

iance (ANOVA); the epoch variable was either searching for a passenger or searching for a store; the region variable was either neocortex-neocortex or neocortex-hippocampus. For the neocortex-neocortex correlation, degrees of freedom were based on correlating each significant theta electrode with all other significant theta electrodes in the neocortex within each patient (e.g., for passenger searching, 855 correlations; and for store searching, 627 correlations). Degrees of freedom for neocortex-hippocampus correlations were based on correlating all significant theta electrodes in hippocampus with all significant theta neocortical electrodes within each patient (e.g., for passenger searching, 92 correlations; for store searching, 36 correlations). We observed a significant effect of epoch $(\mathrm{F}(1,851)=9.1$, MSE $=0.50, P<0.005)$ but no significant effect of region $(\mathrm{F}(1,851)=0.02, \mathrm{MSE}=0.001, P=0.9)$; the interaction was not significant. Our finding of a main effect of epoch shows that theta oscillations were generally less correlated during searching for stores than during searching for passengers.

\section{DISCUSSION}

We set out to test two hypotheses in this work. First, does the human hippocampus, like the human neocortex (e.g., Caplan et al., 2003), show movement-related theta oscillations? Second, can we dissociate hippocampus and neocortex based on the events that trigger movement-related theta? We found movement-related theta oscillations in hippocampus in the raw trace, power spectral density, and $P_{\text {episode }}(f)$ plots for individual electrodes. We further found highly significant numbers of electrodes in hippocampus that showed increased theta oscillations 
during movement but not while standing still. These results, which demonstrate movement-related hippocampal theta, are consistent with previous findings from research on rodents (Vanderwolf, 1969; McFarland et al., 1975; Reece, 1994; Czurko et al., 1999) and from anesthetized nonhuman primates (Stewart and Fox, 1991). Previous studies in humans have demonstrated changes in hippocampal neural firing rate during voluntary movements (Halgren, 1991) and have shown rhythmic slow oscillations in the hippocampus during REM sleep (Bodizs et al., 2001; Cantero et al., 2003). To our knowledge, however, our study is the first to show behavior-related changes in hippocampal theta oscillations in the human.

The second hypothesis we set out to test was whether the presence of movement-related theta during specific tasks allows us to distinguish cortical function from hippocampal function. We found significant numbers of electrodes showing movementrelated theta in disparate regions of cortex (parahippocampal region, temporal, frontal, parietal, and occipital neocortices) as well as in hippocampus. Cortical theta during voluntary movements has been demonstrated in scalp EEG recordings (Klimesch et al., 1994, 1997; Tesche and Karhu, 2000; Klimesch et al., 2001; Babiloni et al., 2004) and during virtual movement in magnetoencephalographic (MEG; see de Araújo et al., 2002) and iEEG recordings (Caplan et al., 2003). A recent study by Mizuhara et al. (2004) using combined functional magnetic resonance imaging (fMRI) and scalp EEG showed coordination of cortical theta between disparate regions (e.g., temporal and frontal cortex) while subjects performed a mental arithmetic task (compared with rest). We typically found high correlation values (explaining on average 20\% of the variance in changes in theta) for electrodes showing movement-related theta both within neocortex and between neocortex and hippocampus. We did not find a significant difference when we compared the mean correlations within neocortex and between neocortex and hippocampus (see Fig. 3). Our findings, together with those of Mizuhara et al. (2004), suggest that bouts of theta tend to be coordinated between disparate brain structures during engaging cognitive tasks, and that theta serves a role in bringing together multiple brain areas for execution of a task. Consistent with the finding of increased theta oscillations during more demanding cognitive tasks (e.g., mental arithmetic compared with rest) by Mizuhara et al. (2004), we also observed greater theta oscillations in neocortex during searching for passengers than during searching for stores. The increased theta oscillations quite likely occurred because searching for passengers required renewed attention during movement so that randomly placed passengers could be located, while finding fixed-location stores did not require the same overall attention from trial to trial because their locations could be learned (Caplan et al., 2003). Although we did not observe significantly greater numbers of electrodes during searching for passengers than during searching for stores in the hippocampus (or hippocampal-parahippocampal combination), the number of significant theta sites and the degree of theta coordination over deliveries did not dissociate hippocampus from the neocortex.
The sensorimotor integration hypothesis (Komisaruk, 1970; Bland, 1986) states that theta oscillations, or neural synchrony, provide constantly updated feedback from sensory regions to voluntary motor regions for correct motor output and performance of a task (Bland and Oddie, 2001). The sensorimotor integration hypothesis therefore argues that theta oscillations should increase based on the attentional demands of a task, and that these changes in theta oscillations should covary between neocortex and hippocampus (Bland and Oddie, 2001). Because we observed increased theta oscillations during movement (and during searching for passengers when compared with searching for stores in the neocortex), and because we could not dissociate changes in theta oscillations between neocortex and hippocampus, our findings support the sensorimotor integration hypothesis. Although our results are derived from a spatial-learning paradigm, other studies have reported increased theta oscillations in disparate cortical sites during nonspatial tasks requiring increased attention (Raghavachari et al., 2001; Sederberg et al., 2003; Mizuhara et al., 2004), suggesting that our conclusions regarding support for the sensorimotor integration hypothesis hold for other cognitive tasks as well.

One potential issue for reconciling our findings with those of Komisaruk (1970) and Bland (1986) is that the increased theta oscillations we observed could be because the subjects are pressing buttons during movement. In our navigation task, however, the subject had to press an arrow key to initiate movement; standing still was marked by release of the key, and to keep moving the subject was required to hold the key down. Because standing-still periods are shorter than movement periods (see Results), the effective rate of key-presses per unit time should be hypothetically greater for still periods. Our findings, however, demonstrated the opposite effect, namely, that more theta was present during movement than while standing still. As further evidence against the explanation that our results are due to button-pressing rather than actual movement, Kahana et al. (1999) and Caplan et al. (2001) observed that shorter mazes had a higher rate of key-presses, yet lower bouts of theta.

Classic work on theta in mammals suggests that different behaviors evoke hippocampal theta and cortical theta. For example, Green and Arduini (1954) found that in mammals (cats, rabbits, and monkeys), changes from drowsiness to alertness were marked by desynchronization in the cortical EEG compared with increasing synchrony in the hippocampal EEG. In contrast, we found that bouts of theta covaried in hippocampus and neocortex during navigation. It is difficult to compare our findings with those of Green and Arduini (1954), because their largely qualitative study did not directly compare theta power in neocortex and hippocampus. It is possible that Green and Arduini did not find these differences because they did not look at active behavior but instead examined changes from sleep to wakefulness. Our finding of no difference between hippocampus and neocortex for which epochs trigger movement-related theta also provides an interesting contrast with single-unit recordings from rats and humans during navigation. For example, the firing rate of rat hippocampal pyramidal cells increases during learning and retrieval of places (e.g., 
O'Keefe and Dostrovsky, 1971), while cells in other brain regions such as the cortex do not show this effect (Jung et al., 1998). Ekstrom et al. (2003) showed that human hippocampal neurons show place-specific firing, while neurons from other regions of the human brain (parahippocampal region, frontal cortex, and amygdala) do not show place specificity. On the basis of these findings, if theta plays a specific role in place learning, one might expect that changes in theta oscillations would be more tightly coupled with learning the locations of fixed-location objects, and that these changes should be specific to the hippocampus. The fact that we did not observe changes in theta oscillations that are specific to spatial learning in the hippocampus strengthens the argument that theta plays a more general role in learning and attention than the firing of single neurons, consistent with the sensorimotor integration hypothesis. The consistency of our EEG recordings with the sensorimotor integration hypothesis, coupled with our previous finding of a dissociation between hippocampus and other brain regions for place learning at the single neuron level (Ekstrom et al., 2003), strengthens the argument that oscillations, and oscillatory synchrony, relate to the activation and coordination of disparate brain regions involved in performing a task. Neural firing rate, in contrast, may relate more closely to the actual computational function of a structure.

\section{REFERENCES}

Babiloni C, Babiloni F, Carducci F, Cappa SF, Cincotti F, Del Percio C, Miniussi C, Vito Moretti D, Rossi S, Sosta K, Rossini PM. 2004. Human cortical rhythms during visual delayed choice reaction time tasks. A high-resolution EEG study on normal aging. Behav Brain Res 153:261-271.

Berry SD, Seager MA. 2001. Hippocampal theta oscillations and classical conditioning. Neurobiol Learn Mem 76:298-313.

Bland BH. 1986. The physiology and pharmacology of hippocampal formation theta rhythms. Prog Neurobiol 26:1-54.

Bland BH, Colom LV. 1993. Extrinsic and intrinsic properties underlying oscillation and synchrony in limbic cortex. Prog Neurobiol 41:157-208.

Bland BH, Oddie SD. 2001. Theta band oscillation and synchrony in the hippocampal formation and associated structures: the case for its role in sensorimotor integration. Behav Brain Res 127:119-136.

Bodizs R, Kantor S, Szabo G, Szucs A, Eross L, Halasz P. 2001. Rhythmic hippocampal slow oscillation characterizes REM sleep in humans. Hippocampus 11:747-753.

Cameron K, Yashar S, Wilson C, Fried I. 2001. Human hippocampal neurons predict how well word pairs will be remembered. Neuron 30:289-298.

Cantero JL, Atienza M, Stickgold R, Kahana MJ, Madsen JR, Kocsis B. 2003. Sleep-dependent theta oscillations in the human hippocampus and neocortex. J Neurosci 23:10897-10903.

Caplan JB, Madsen JR, Raghavachari S, Kahana MJ. 2001. Distinct patterns of brain oscillations underlie two basic parameters of human maze learning. J Neurophys 86:368-380.

Caplan JB, Madsen JR, Schulze-Bonhage A, Aschenbrenner-Scheibe R, Kahana MJ. 2003. Human theta oscillations related to sensorimotor integration and spatial learning. J Neurosci 23:4726-4736.

Czurko A, Hirase H, Csicsvari J, Buzsaki G. 1999. Sustained activation of hippocampal pyramidal cells by space clamping in a running wheel. Eur J Neurosci 11:345-352. de Araújo DB, Baffa O, Wakai RT. 2002. Theta oscillations and human navigation: a magnetoencephalography study. J Cogn Neurosci 14:70-78.

Ekstrom AD, Meltzer J, McNaughton BL, Barnes CA. 2001. NMDA receptor antagonism blocks experience-dependent expansion of hippocampal place fields. Neuron 31:631-638.

Ekstrom AD, Kahana MJ, Caplan JB, Fields T, Isham E, Newman E, Fried I. 2003. Cellular networks underlying human spatial navigation. Nature 425:184-187.

Fried I, MacDonald K, Wilson C. 1997. Single neuron activity in human hippocampus and amygdala during recognition of faces and objects. Neuron 18:753-765.

Fried I, Wilson C, Maidment N, Engel J, Behnke E, Fields T, MacDonald K, Morrow J. 1999. Cerebral microdialysis combined with single-neuron and electroencephalographic recording in neurosurgical patients. J Neurosurg 91:697-705.

Green JD, Arduini AA. 1954. Hippocampal electrical activity in arousal. J Neurophys 17:533-557.

Grossmann A, Morlet J. 1985. Decomposition of functions into wavelets of constant shape, and related transforms. In: Streit L, editor. Mathematics + physics. Vol 1. Singapore: World Scientific. p 135165.

Halgren E. 1991. Firing of human hippocampal units in relation to voluntary movements. Hippocampus 1:153-161.

Jung MW, Qin Y, McNaughton BL, Barnes CA. 1998. Firing characteristics of deep layer neurons in prefrontal cortex in rats performing spatial working memory tasks. Cerebr Cortex 8:437-450.

Kahana MJ, Sekuler R, Caplan JB, Kirschen MP, Madsen JR. 1999. Human theta oscillations exhibit task dependence during virtual maze navigation. Nature 399:781-784.

Kamondi A, Acsady L, Wang XJ, Buzsaki G. 1998. Theta oscillations in somata and dendrites of rat hippocampal pyramidal cells in vivo: activity-dependent phase-precession of action potentials. Hippocampus 8:244-261.

Klimesch W, Schimke H, Schwaiger J. 1994. Episodic and semantic memory: an analysis in the EEG theta band. Electroencephalogr Clin Neurophysiol 91:428-441.

Klimesch W, Doppelmayr M, Schimke H, Ripper B. 1997. Theta synchronization and alpha desynchronization in a memory task. Psychophysiology 34:169-176.

Klimesch W, Doppelmayr M, Yonelinas A, Kroll NE, Lazzara M, Rohm D, Gruber W. 2001. Theta synchronization during episodic retrieval: neural correlates of conscious awareness. Brain Res Cogn Brain Res 12:33-38.

Komisaruk BR. 1970. Synchrony between limbic system theta activity and rhythmical behavior in rats. J Comp Physiol Psychol 70:482492.

Leutgeb S, Mizumori SJ. 1999. Excitotoxic septal lesions result in spatial memory deficits and altered flexibility of hippocampal singleunit representations. J Neurosci 19:6661-6672.

McFarland W, Teitelbaum H, Hedges E. 1975. Relationship between hippocampal theta activity and running speed in the rat. J Comp Physiol Psychol 88:324-328.

Mehta MR, Lee AK, Wilson MA. 2002. Role of experience and oscillations in transforming a rate code into a temporal code. Nature 417:741-746.

Mizuhara H, Wang LQ, Kobayashi K, Yamaguchi Y. 2004. A longrange cortical network emerging with theta oscillation in a mental task. Neuroreport 15:1233-1238.

Niedermeyer E, Lopes da Silva F. 1999. Electroencephalography of the walking adult. Philadelphia: Lippincott Williams and Wilkins.

O'Keefe J, Dostrovsky J. 1971. The hippocampus as a spatial map. Preliminary evidence from unit activity in the freely moving rat. Brain Res 34:171-175.

O’Keefe J, Reece ML. 1993. Phase relationship between hippocampal place units and the EEG theta rhythm. Hippocampus 3:317-330. 
Percival DB, Walden AT. 1993. Spectral analysis for physical applications: multitaper and conventional univariate techniques. Cambridge: Cambridge University Press.

Raghavachari S, Rizzuto D, Caplan J, Kirschen M, Bourgeois B, Madsen J, Kahana MJ, Lisman J. 2001. Gating of human theta oscillations by a working memory task. J Neurosci 21:3175-3183.

Reece M. 1994. The representation of space in the rat hippocampus as revealed using new computer-based methods. London: University College of London. Unpublished dissertation.

Sederberg PB, Kahana MJ, Howard MW, Donner EJ, Madsen JR. 2003. Theta and gamma oscillations during encoding predict subsequent recall. J Neurosci 23:10809-10814.
Shen J, Barnes CA, McNaughton BL, Skaggs WE, Weaver KL. 1997. The effect of aging on experience-dependent plasticity of hippocampal place cells. J Neurosci 17:6769-6782.

Stewart M, Fox SE. 1991. Hippocampal theta activities in monkeys. Brain Res 538:59-63.

Tesche C, Karhu J. 2000. Theta oscillations index human hippocampal activation during a working memory task. Proc Nat Acad Sci USA 97:919-924.

Vanderwolf CH. 1969. Hippocampal electrical activity and voluntary movement of the rat. Electroencephalogr Clin Neurophysiol 26: 407-418.

Witter M, Wouterlood F. 2002. The parahippocampal region: past, present, and future. Oxford: Oxford University Press. 